\title{
The role of species charisma in biological invasions
}

Ivan Jaric $^{1 *}$, Franck Courchamp ${ }^{2}$, Ricardo A Correia ${ }^{3}$, Sarah L Crowley $^{4}$, Franz Essl ${ }^{5}$, Anke Fischer ${ }^{6}$, Pablo González-Moreno ${ }^{7,8}$, Gregor Kalinkat $^{9}$, Xavier Lambin ${ }^{10}$, Bernd Lenzner ${ }^{5}$, Yves Meinard ${ }^{11}$, Aileen Mill ${ }^{12}$, Camille Musseau ${ }^{9,13}$, Ana Novoa ${ }^{14}$, Jan Pergl ${ }^{14}$, Petr Pyšek ${ }^{14,15}$, Klára Pyšková ${ }^{14,15}$, Peter Robertson ${ }^{12}$, Menja von Schmalensee ${ }^{16}$, Ross T Shackleton ${ }^{17}$, Robert A Stefansson ${ }^{16}$, Kateřina Štajerová ${ }^{14,15}$, Diogo Veríssimo ${ }^{18,19}$, and Jonathan M Jeschke,13

Commonly used in the literature to refer to the "attractiveness", "appeal", or "beauty" of a species, charisma can be defined as a set of characteristics - and the perception thereof - that affect people's attitudes and behaviors toward a species. It is a highly relevant concept for invasion science, with implications across all stages of the invasion process. However, the concept of invasive alien species (IAS) charisma has not yet been systematically investigated. We discuss this concept in detail, provide a set of recommendations for further research, and highlight management implications. We review how charisma affects the processes associated with biological invasions and IAS management, including species introductions and spread, media portrayals, public perceptions of species management, research attention, and active public involvement in research and management. Explicit consideration of IAS charisma is critical for understanding the factors that shape people's attitudes toward particular species, planning management measures and strategies, and implementing a combination of education programs, awareness raising, and public involvement campaigns.

Front Ecol Environ 2020; 18(6):345-353, doi:10.1002/fee.2195

The concept of charismatic species - commonly used in the scholarly literature to refer to the "attractiveness", "appeal", or "beauty" of a given species (Panel 1) - has recently garnered attention in conservation science due to its potential to stimulate public awareness and support,

\section{In a nutshell:}

- The charisma of invasive alien species (IAS) is an important concept for invasion science, but it has not yet been properly addressed in the literature

- We explore IAS charisma in terms of invasion dynamics and management, and provide a set of recommendations for further research

- The charisma of IAS can influence all stages of the invasion process, and both charisma and its influence tend to vary over time and space

- IAS charisma can affect management actions by influencing public support or contributing to social conflicts

- Charisma can, and historically has affected species introductions, media portrayal, public perceptions, opposition to management, research effort, and public participation in research and management

${ }^{1}$ Biology Centre of the Czech Academy of Sciences, Institute of Hydrobiology, České Budějovice, Czech Republic

*(ivan.jaric@hbu.cas.cz); ${ }^{2}$ Université Paris-Saclay, CNRS, AgroParisTech, Ecologie Systématique Evolution, Orsay, France;

${ }^{3}$ Helsinki Lab of Interdisciplinary Conservation Science,

Department of Geosciences and Geography, (continued on last page) especially through the use of flagship species (Veríssimo et al. 2011; Courchamp et al. 2018). The charisma of any introduced species, and invasive alien species (IAS) in particular, can affect people's perceptions and attitudes toward management of that species (McNeely 2001; Veitch and Clout 2001; Shackleton et al. 2019). Research demonstrates how IAS charisma can influence the invasion process across a wide range of organisms spanning different taxonomic groups and regions (WebTables 1-3; Figure 1). Unlike the charisma of threatened species, which has a positive effect on management efforts, charisma in IAS usually represents a hindrance to management (Genovesi and Bertolino 2001; Bertolino and Genovesi 2003). Charisma can reduce public support for IAS management attempts and contribute to conflicting perceptions and interests, and ultimately impede management efforts (eg by delaying or preventing control implementation; Estévez et al. 2015; Novoa et al. 2018). However, the issue of species charisma in relation to IAS has not yet been systematically explored.

We discuss the concept of species charisma in the context of IAS, and explore how it can affect species introductions, media portrayals, public perceptions, opposition to management, research efforts, and public participation in research and management (Figure 1). In addition to clarifying the concept of charismatic IAS (Panel 1), we illustrate how the perception of charisma is highly context-dependent and varies over space and time. Identifying these issues enables us to provide a set of recommendations for further research, and to highlight both management implications and measures that can be taken to address this issue. 


\section{Panel 1. Invasive alien species (IAS) charisma}

Species charisma is a highly complex concept, and there is currently no consensus on a definition (Lorimer 2007; Albert et al. 2018). It is used in the literature to refer to the "attractiveness", "appeal", or "beauty" of a species but, with the exception of the seminal work by Lorimer (2007), very few studies have actually stated what the term signifies or what its properties are (Albert et al. 2018; Crowley et al. 2019). Lorimer (2007) refers to species charisma as "non-human charisma" and defines it as a concept that lies somewhere between inherent species characteristics on the one hand and subjective perceptions and values assigned by humans on the other, with the latter generated through direct or indirect human interactions with the species (Crowley et al. 2019). Being highly subjective, perceptions of charisma can change over time, or even be enhanced or constructed by marketing campaigns or the media (Lorimer 2007). This author further pointed out that charisma is not always unambiguously positive; for example, species can be perceived as both charismatic and frightening (eg sharks, large snakes). In wider use, however, and particularly in conservation, the term is applied to those species whose characteristics and behavior tend to inspire positive responses in humans. In conservation science and practice, charisma is closely associated with the flagship species concept, and used for scientific communication and for attracting funds (Albert et al. 2018).

Our definition of charismatic species, and of charismatic invasive alien species (IAS) in particular, therefore relates to those species whose characteristics affect people's perceptions, attitudes, and behaviors toward them.
We refer here to the behavior of both management activities by institutions and reactions by society in general. Characteristics that drive species charisma can be visual (eg unique morphology), acoustic (particular sounds produced, such as bird calls), olfactory (emission of pleasant smells; for instance, the fragrances produced by flowering plants), behavioral (eg complex or anthropomorphic behavior), or symbolic (abstract characteristics embedded in the general culture). Nevertheless, species charisma is highly context dependent; varies over space and time; and is influenced by regional, social, and cultural factors, as well as individual value systems (Shackleton et al. 2019). For instance, people can have notably different perceptions of squirrels, either considering them charismatic due to their features (eg large eyes, bushy tails) or loathing their rodent-like characteristics (Shackleton et al. 2019). A detailed overview of factors influencing human perceptions of IAS was provided by Shackleton et al. (2019).

Although the definition of species charisma is elusive, some animal traits are known to contribute to charisma, including body size, distinctive coloration, furry coat, peculiar appearance, neotenic (juvenile) features, and sentience (Gobster 2011; Shackleton et al. 2019; Beever et al. 2019). Feral populations of domestic animals (eg feral cats and dogs, wild horses) are especially likely to be charismatic (Veitch and Clout 2001). Some plants can also be charismatic due to traits such as foliage shape and flower color, size, and fragrance (Mack 2001; Veitch and Clout 2001; Shackleton et al. 2019).

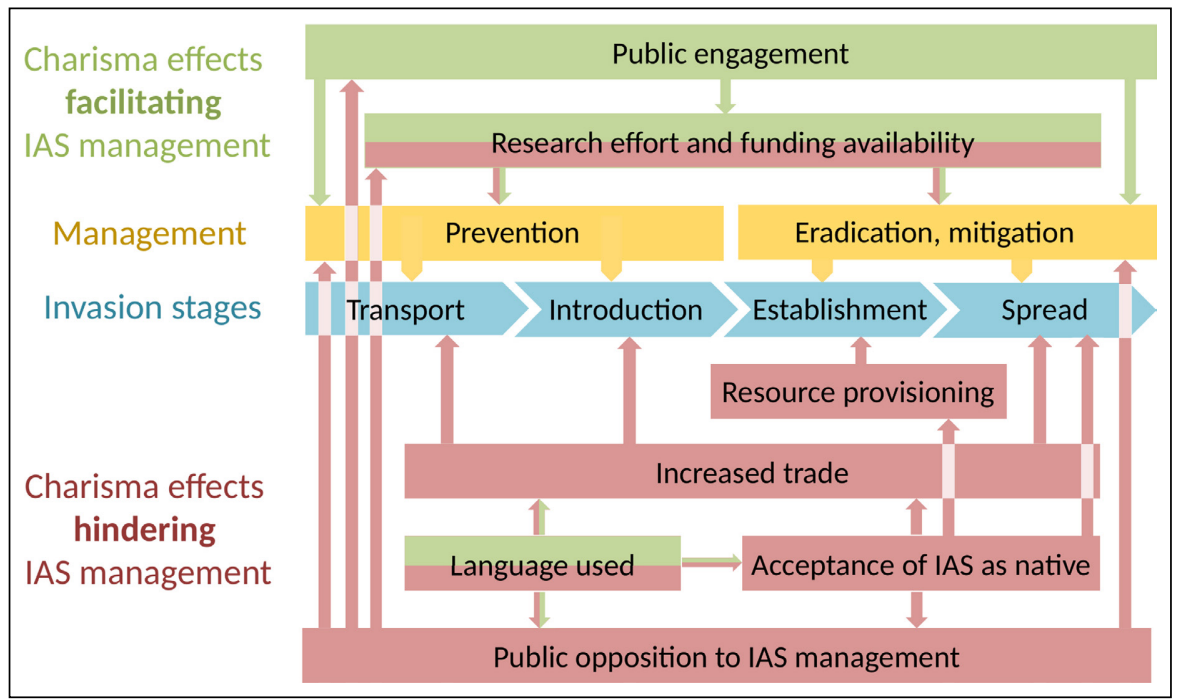

Figure 1. Overview of different mechanisms through which invasive alien species (IAS) charisma affects different invasion stages and management measures. Invasion stages are based on the framework by Blackburn et al. (2011). Red fields and arrows represent charisma effects that tend to hinder IAS management; green fields and arrows represent charisma effects that tend to facilitate IAS management; and bicolored fields and arrows represent charisma effects that can either hinder or facilitate IAS management, depending on the circumstances.

\section{Effects of charisma on introductions and establishment success}

Charisma likely affects introduction and establishment rates, especially for certain taxonomic groups and introduction pathways, such as the ornamental plant, aquarium, and pet trades (Padilla and Williams 2004; van Kleunen et al. 2018). For example, aquarium releases are recognized as a key contemporary introduction pathway for invasive aquatic species, and are responsible for the introduction of as much as one-third of the world's ecologically and economically most damaging aquatic IAS (Padilla and Williams 2004). Such aquatic ornamental species (along with their terrestrial counterparts) are not randomly selected, but instead are chosen for specific, appealing traits, resulting in higher demand for charismatic species in the pet and horticultural trades (Chucholl and Wendler 2017; van Kleunen et al. 2018; Kutlvašr et al. 2019). The increased prominence of charismatic organisms within the pool of traded and reared species will likely facilitate their representation within the pool of introduced species and may consequently increase propagule pressure (ie frequency and size of introduction events), although this remains to be quantified. Charisma probably has negligible effects on inadvertent introductions (eg via ballast water or seed contamination) or those mainly driven by perceived utility (eg crop species), but we postulate that charismatic species should have a comparatively higher overall chance 
of being introduced, all else being equal, than non-charismatic species. If this hypothesis is confirmed, then species charisma could potentially shape the composition of the introduced species pool, and charismatic species would also be more likely to become established than non-charismatic species. Indeed, some of the best-known IAS introductions were likely influenced by their charisma. The raccoon (Procyon lotor) is a good example; they are very charismatic due to their "cute" appearance, with facial color patterns that resemble a bandit's mask, and behavioral traits that are perceived as comical, such as their endearing habit of dousing, or supposedly washing food prior to eating. Because of this, the raccoon became a very popular pet in Japan - where it is an alien species - and many individuals were imported; it has since spread across the country (Ikeda et al. 2004). Other examples are presented in Figure 2 and WebTables 1-3. However, what constitutes charisma is dynamic, changing over time and differing among cultures. This limits the predictive power of analyses of future charismadriven invasions based on historical events.

Charisma can also have a strong effect on the establishment success of introduced species, through public support and active provisioning of resources. Typical examples include winter-food provisioning for charismatic alien birds via bird feeders (Crowley et al. 2019), and feeding of feral domestic cats (Felis catus) and dogs (Canis familiaris) (Allen 2018).
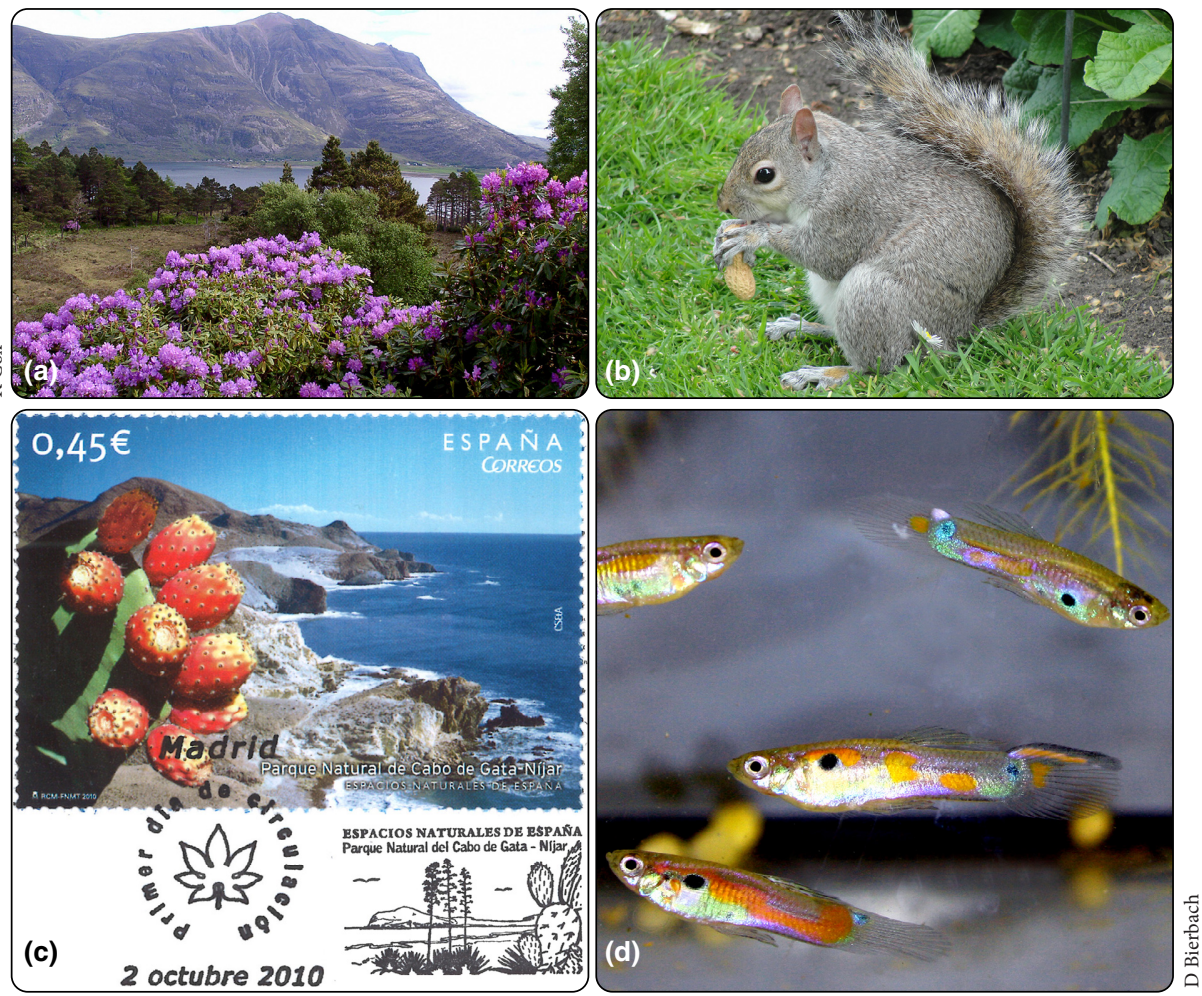

Figure 2. Examples of IAS charisma effects on biological invasions and management measures. (a) Introduction rates: introduction of pontic rhododendron (Rhododendron ponticum) was predominantly driven by its charisma (Mack 2001). (b) IAS charisma gives rise to public opposition to control measures: proposed control measures for introduced eastern gray squirrel (Sciurus carolinensis) populations in Italy were delayed and made ineffective by strong public opposition (Bertolino and Genovesi 2003). (c) IAS charisma contributes to societal acceptance of IAS: Opuntia species in Spain have become iconic symbols in the landscape, and have been depicted on stamps and postmarks. (d) IAS charisma can contribute to volunteer involvement in citizen-science projects: guppies (Poecilia reticulata) were promoted as flagship species of a citizen-science project directed at monitoring alien fish species in thermally polluted waters in Germany (Lukas et al. 2017).
Some species traits contribute to both the establishment potential of a species and the likelihood that it will be perceived as charismatic, which makes them especially relevant for invasion risk assessments. For ornamental plants, long flowering periods, multiple flowering events, and height are particularly desirable, and are also traits that are positively associated with establishment success and invasiveness (Pyšek and Richardson 2008; van Kleunen et al. 2018). Similarly, many of the alien bird species that were successfully introduced by acclimatization societies (voluntary associations that promoted introductions of alien species for scientific, economic, leisure, or aesthetic purposes) in the 19th and 20th centuries were characterized by a combination of appealing features and traits that facilitated their establishment and spread.

\section{Effects of IAS charisma on media and communication}

In addition to direct experience with IAS impacts, public awareness and perception of IAS can stem from indirect sources of information, such as the media, and charisma has a tendency to affect the style and tone of language used by media outlets (Veitch and Clout 2001; Larson 2005). Media portrayals are more likely to feature either charismatic species or those with serious environmental or economic impacts (Veitch and Clout 2001; Wilson et al. 2007; Jarić et al. 2019). The perception of species charisma can therefore be influenced (both positively and negatively) by the way species are portrayed, via increased media exposure or by emphasizing specific points of view (Figure 3; Veitch and Clout 2001; Crowley et al. 2017; Shackleton et al. 2019). As a case in point, public perception and the stance of official bodies toward Nootka lupine (Lupinus nootkatensis) in Iceland shifted from positive to largely negative because of an ongoing public debate in the media (Petursdottir et al. 2013; Benediktsson 2015). Effects of IAS charisma and their media representation and communication are essentially interrelated, in that they influence each other and the way species will be ultimately perceived. 

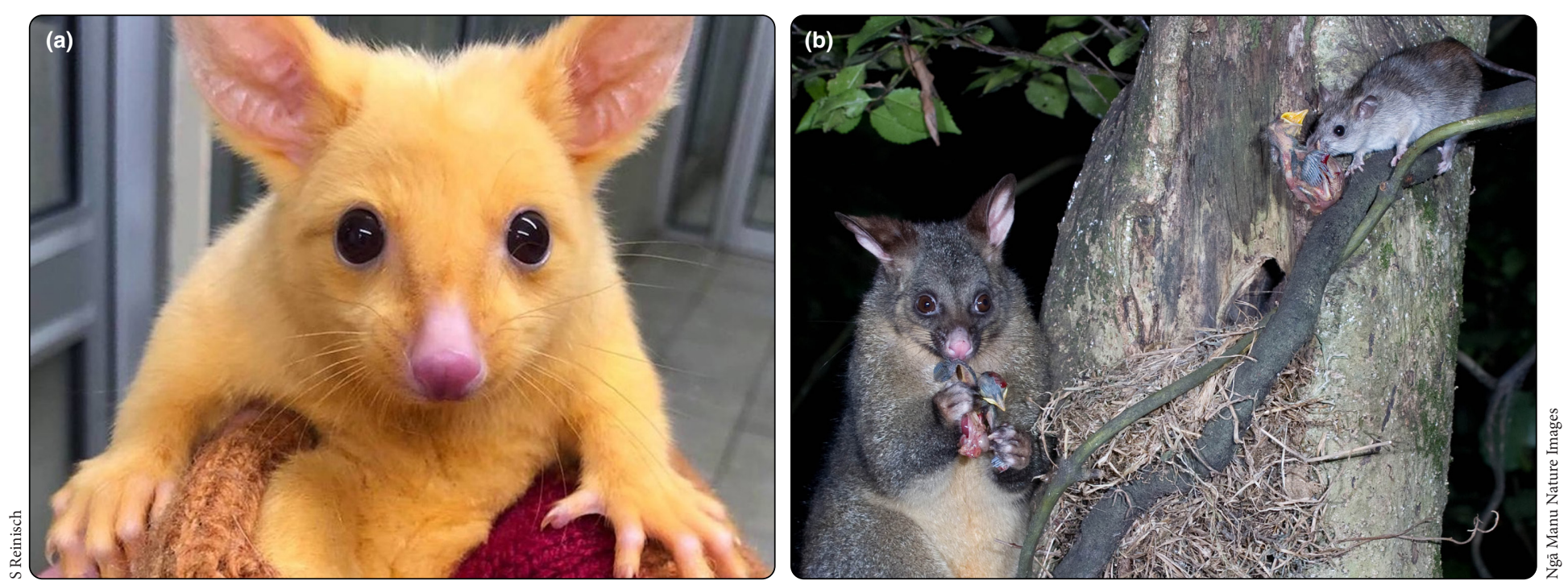

Figure 3. The way in which an IAS is presented in the media can strongly affect public perception. An example includes the common brushtail possum (Trichosurus vulpecula), which has been depicted both (a) positively as a "Pikachu"-like animal and (b) negatively, here with a black rat (Rattus rattus) as nest predators of the song thrush (Turdus philomelos). All three species are IAS in New Zealand.

Messages emphasized with emotive language may result in partial reporting and public misinformation (Crowley et al. 2019). For example, newspapers and animal rights groups in Italy used emotive messages in articles about a planned control program for the invasive eastern gray squirrel (Sciurus carolinensis; Figure 2b) by depicting squirrels in the form of endearing cartoon characters, which affected public perception and attitude toward the species (Genovesi and Bertolino 2001). This led to protracted legal proceedings and a subsequent withdrawal of funding, thereby contributing to the failure of the eradication campaign (Genovesi and Bertolino 2001; Shackleton et al. 2019). Management of some IAS can be associated with intense conflicts, where various stakeholders (eg journalists, scientists, resource managers, government institutions, non-governmental organizations) have frequently resorted to militaristic language and bellicose metaphors to pursue and advocate for desired research and management activities (Larson 2005; Wallach et al. 2018). Stakeholders' perceptions of IAS charisma may influence the way such conflicts emerge or escalate, and the manner in which these perceptions are communicated could in turn also affect the perceived charisma (eg by referring to a plant species as a weed or an animal species as a pest). A good example is Echium plantagineum, a European herb introduced into Australia, which is called "salvation Jane" in South Australia and "Patterson's curse" elsewhere in the country (Kueffer and Kull 2017). While the name reflects how the species tends to be perceived regionally (ie as either a useful crop or a noxious weed), the choice of name can also affect public perception of the plant.

\section{Effects of charisma on societal acceptance of IAS}

Perceptions of the natural state of the environment are to a large extent socially constructed and context-dependent
(Backstrom et al. 2018). Public attitudes toward a species can be influenced by its origin but other factors are usually more important, such as economic value and impact (van der Wal et al. 2015) or charisma (Gobster 2011; WebTables $1-3)$; for instance, large trees are often valued by the public regardless of their origin (Gobster 2011).

IAS may become accepted by the public as desirable elements of local fauna and flora, and as such often serve as examples of shifting baseline syndrome (a gradual change in accepted norms due to a lack of experience, memory, or knowledge; Soga and Gaston 2018; Beever et al. 2019). Over time, expectations about what is a truly original and desirable state of the natural environment evolve (Soga and Gaston 2018), and people's ability to recognize a species as alien decreases as the time since introduction increases (GarciaLlorente et al. 2008).

Public acceptance of an alien species likely increases with the perceived charisma of the species, especially if it is associated with cultural practices or perceptions of the local environment (Nuñez and Simberloff 2005; Verbrugge et al. 2013). For example, after introduction into southeast Spain for economic reasons, Agave and Opuntia species became widespread in the country's arid areas. As a result of being increasingly perceived as charismatic, over time they came to be recognized as iconic symbols of the regional landscape, to the point where they have even been depicted on stamps and postmarks (Figure 2c). Similarly, Jacaranda trees are so iconic in South Africa that they are now a symbol of Pretoria, which came to be known as the "Jacaranda City" (Dickie et al. 2014). Alien species can be integrated into cultural identities through positive interactions and emotional and material attachments, and such processes can occur relatively quickly in the case of charismatic species (Crowley et al. 2017, 2018). In Chicago, monk parakeets (Myiopsitta monachus) have become an iconic species within 


\section{Panel 2. The case of feral hippopotamuses in Colombia}

A small population of feral hippopotamuses (Hippopotamus amphibius) currently lives in the Rio Magdalena valley in northeastern Colombia (Figure 4), and is a case of a charismatic IAS that has garnered a considerable amount of international media attention. Drug cartel leader Pablo Escobar illegally imported four hippopotamuses for the establishment of a private zoo on his estate in the early 1980s, but after his death in 1993 and subsequent forfeiture of his estate, they were allowed to roam the surrounding countryside and have been reproducing successfully ever since, with potential negative impacts on native ecosystems (Dembitzer 2017). There have been several unsuccessful attempts to control the growth of the population, which is currently estimated to consist of up to 70 individuals. Culling initiatives were abandoned due to strong public opposition in 2009, and sterilization plans have been cancelled due to high costs and the risks to both the hippopotamuses and humans during the procedure. Hippopotamuses are among the most charismatic animal species because of their impressive body size and appearance (Albert et al. 2018), and they are valued by local communities, as it is believed that they attract tourists to the area; they are now increasingly featured in decorative motifs in public spaces and commercial enterprises.

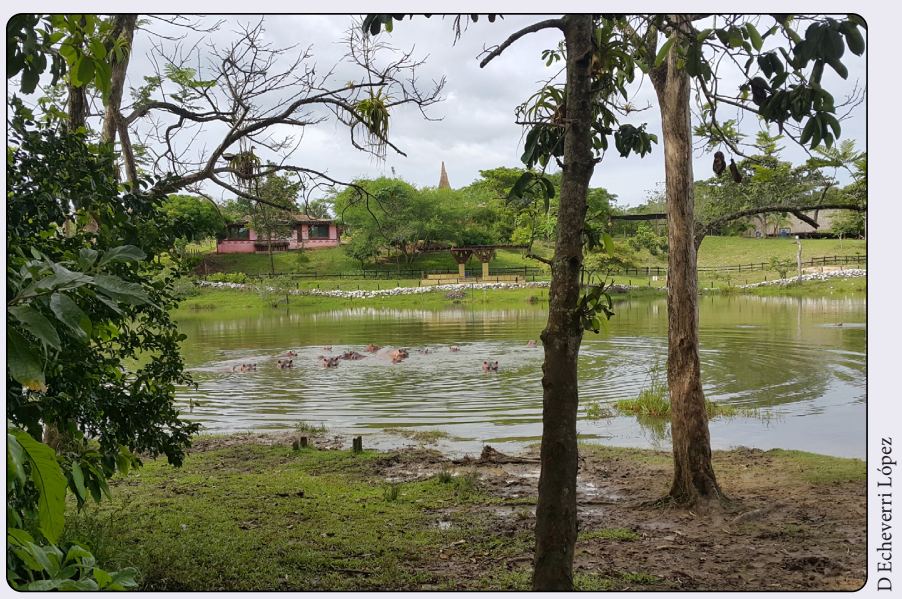

Figure 4. Feral hippopotamuses (Hippopotamus amphibius) in the Rio Magdalena, northeastern Colombia. less than 50 years after their introduction (Crowley et al. 2017), and the ruddy duck (Oxyura jamaicensis) has been adopted as the emblem of the birdwatchers' club in the region of the UK where it was first introduced. Many alien species are nowadays considered desirable and might even be subject to protection or restoration measures in situations where they are threatened or suffer population declines (Clavero 2014; Crowley et al. 2018). In some cases, charismatic IAS can provide economic benefits (eg through tourism), which further promotes their societal acceptance (Panel 2).

\section{Effects of charisma on the likelihood of public opposition to IAS management}

A lack of public support for, or even opposition to, IAS management is not uncommon (Crowley et al. 2017; Novoa et al. 2018), and can also be influenced by IAS charisma (Fischer et al. 2014). Plans to control species perceived as charismatic have often faced opposition, whereas no such resistance is the norm for species that are not perceived as charismatic (with the exception of those that provide economic benefits; Liordos et al. 2017). Some well-known instances where public opposition has hindered IAS control due to perceived charisma include invasive populations of monk parakeet and mute swan (Cygnus olor) in the US, and hippopotamus (Hippopotamus amphibius) in Colombia (Panel 2; Ellis and Elphick 2007; Dembitzer 2017; Crowley et al. 2019). Opposition also frequently arises in response to attempts to control feral populations of charismatic pets and domestic animals, such as cats, dogs, and horses (Equus caballus) (Veitch and Clout 2001; Estévez et al. 2015; Allen 2018). Due to a strong taxonomic bias in perceptions of charisma, public opposition to the control of invasive mammal or bird species is more likely to occur than against control of invertebrates or plants (Shackleton et al. 2019). However, attempts to control charismatic alien plants, such as large pines (Pinus spp) or eucalypti (Eucalyptus spp), have also faced opposition (Nuñez and Simberloff 2005; Dickie et al. 2014; Estévez et al. 2015).

There also appears to be a relationship between species charisma and a public consensus on the acceptability of particular control measures - that is, there is often less acceptance of direct and lethal control methods for the more charismatic species (Verbrugge et al. 2013; Fischer et al. 2014). Opposition by vocal sections of the public has at times forced management authorities to implement alternative, non-lethal, and often more expensive methods of control, such as relocation or reproduction suppression, despite such measures often being less effective (Panel 2; Bertolino and Genovesi 2003; Verbrugge et al. 2013).

\section{Effects of IAS charisma on research efforts and funding availability}

Invasion science is taxonomically biased, and only a minority of IAS are studied in detail (Wilson et al. 2007; Pyšek et al. 2008). While the taxonomic focus is largely driven by IAS impacts (Pyšek et al. 2008), there is, overall, a greater focus on invasive vertebrates than invertebrates, and on large and charismatic species (Wilson et al. 2007). Research biases can lead to knowledge gaps, and may negatively affect conservation prioritization, management effectiveness, international decision making, and policy development (Donaldson et al. 2016). 


\section{Panel 3. Potential of the flagship species concept in IAS management}

The concept of "flagship species" was initially developed to focus conservation marketing campaigns on species with traits that are perceived as charismatic, with the intent of attracting public support and funding for conservation efforts (eg giant panda, Ailuropoda melanoleuca; Veríssimo et al. 2011). However, IAS charisma will in most cases constrain management of such species by diminishing support for control measures.

One use of charismatic IAS as flagship species that can be beneficial is for monitoring programs and citizen-science initiatives, as they can help to motivate volunteers to become engaged in sampling or monitoring activities (Figure 2d; Lukas et al. 2017).

Arguably, the most promising way to apply the flagship species concept in IAS management is to focus on any charismatic species that are impacted by IAS. Such conservation marketing campaigns can highlight either the species threatened by IAS, or selected species pairs, represented by the IAS and its charismatic victim. The charisma of the "flagship victim" can potentially mitigate effects of IAS charisma on public support for management, and this concept has already been adopted in several local IAS management actions. Examples include the endangered southern cassowary (Casuarius casuarius) as a flagship victim of wild boars (Sus scrofa) in Queensland, Australia (McNeely 2001); the SOS Puffin project in the Firth of Forth, Scotland, where the Atlantic puffin (Fratercula arctica) is portrayed as a flagship victim of the invasive mallow tree (Lavatera arborea; Pagès et al. 2018); and the water vole (Arvicola amphibius), which is depicted as a flagship victim of American mink (Neovison vison) in Scotland (Melero 2017).
IAS charisma may also affect research efforts, with charismatic IAS receiving more research interest (eg through personal preferences of individual researchers or potentially greater availability of funding). Although this has not been directly examined in the field of invasion science, there is a well-established effect of charisma in conservation science (Clark and May 2002; Fleming and Bateman 2016; Jarić et al. 2019). Moreover, the social sciences and humanities are interested in both individual and social processes, such as discourses, attitudes, behavioral changes, and conflicts (Schüttler et al. 2011). Such societal dynamics are more likely to arise from charismatic IAS, and are therefore more likely to lead to comparatively greater research effort being focused on sociocultural aspects of charismatic IAS. At the same time, applied research can also be impeded by reduced funding support and public opposition to management, such as in the case of the eastern gray squirrel in Italy, where public opposition obstructed a pilot research project geared toward squirrel eradication (Genovesi and Bertolino 2001).

\section{Effects of IAS charisma on active public involvement in research and management}

Volunteer initiatives are increasingly recognized as an affordable tool for managing biological invasions (Pagès et al. 2018). However, public involvement in controlling highly charismatic species may be limited, at least in some cases (Crowley et al. 2018). Unappealing appearance or behavioral traits and negative perception of a species can be more beneficial for control efforts. The introduced cane toad (Rhinella marina) in Australia is one such example, in which strong public aversion to this species attracted substantial volunteer participation in various management activities (Estévez et al. 2015).

Nevertheless, IAS charisma can often have potentially positive effects, for example by motivating the public to actively engage in hunting, fishing, or other public initiatives directed at invaders perceived as attractive game species
(Green et al. 2017). Annual hunting derbies directed at charismatic invasive Indo-Pacific lionfish (Pterois spp) in the Western Atlantic have attracted considerable volunteer effort and are effective for suppression of local lionfish populations (Green et al. 2017). Several traits that contribute to species charisma may simultaneously make them easier to detect (eg bright colors, large body size, unique morphology), thereby increasing the efficiency of monitoring programs and citizen-science initiatives. However, management initiatives based on public promotion of IAS charisma must be evaluated against potential associated risks, such as promotion of further invasions, incorporation of such species into local cultures (Nuñez et al. 2012), and encouragement of public engagement that may also target threatened native species.

IAS charisma can also motivate the active involvement of specific groups (eg aquarium hobbyists) to contribute to scientific research, education, and raising awareness, as well as to campaigns on IAS trade and introduction control (MacedaVeiga et al. 2016). In some cases, it can also stimulate public involvement through the use of the flagship species concept (Panel 3).

\section{Conclusions}

If the extent of human contributions to biological invasions, as well as management successes and failures, are to be fully understood, it is critical to explicitly recognize the importance of charisma associated with IAS (WebFigure 1). Interventions aimed at changing attitudes and behaviors toward charismatic IAS, and to raise awareness of their potential impacts, can reduce risks arising from the trade and cultivation of invasive species and their introductions. They can also bolster support for control measures and volunteer participation in management initiatives. Perception of species charisma is highly context- and culture-dependent (Lorimer 2007), and can be modified through targeted activities (Panel 1; Veríssimo et al. 2017). Several conservationists 
have advocated behavior change interventions, a set of techniques developed to influence people's choices in ways that will positively affect the environment (Byerly et al. 2018). In addition to behavioral changes, these types of strategies can also be used to influence attitudes toward IAS and perceptions of charisma.

Open communication, improved collaboration, and engagement among scientists, managers, and key stakeholders can greatly reduce the risk of conflicts, and foster establishment of joint management goals and initiatives (Fischer et al. 2014; Crowley et al. 2017; Novoa et al. 2018). Conflicts, especially those associated with charismatic IAS, often stem from the apparent incompatibility of differing ethical perspectives: for instance between those prioritizing ecosystem health or species conservation and those concerned for the welfare of the alien species (Genovesi and Bertolino 2001; Wallach et al. 2018).

To better ensure positive conservation outcomes, resource managers must acknowledge and anticipate public perceptions toward IAS, as well as consider the power of charisma in the planning and implementation of management actions. Any effects of IAS charisma on different facets of human well-being should also be defined and integrated within established frameworks for socioeconomic impact classification (Bacher et al. 2018). Although measuring species charisma is challenging due to subjectivity and the instability of societal charisma perceptions (Panel 1), future studies should try to address this issue. Improved understanding of IAS charisma will require careful consideration of values, perceptions, and the cultural background of different stakeholders, as well as of cultural trends and variability (Garcia-Llorente et al. 2008; Crowley et al. 2017). Research based on social-science methods will be key to developing a better understanding of IAS characteristics, societal values, and other factors that give rise to IAS charisma. Digital approaches involving analysis of large bodies of text and other media represent potentially valuable additional research tools for exploring human cultures, identifying key traits and drivers of IAS charisma, and understanding and monitoring public perceptions of IAS and their trends over space and time (Ladle et al. 2016).

\section{Acknowledgements}

We thank DL Strayer for valuable comments and suggestions, as well as L Akins, B Allen, D Bierbach, J Brejcha, K Cox, D Echeverri López, S Green, D Hall, M Rueda, MA Young, R Mills, Ngā Manu Nature Reserve, S Reinisch, and the Boronia Veterinary Clinic and Animal Hospital for providing photographs. This work was supported by the JE Purkyně Fellowship (Czech Academy of Sciences) and Alexander von Humboldt Foundation (IJ); EXPRO grant SynHab \#19-28807X (Czech Science Foundation); long-term research development project RVO 67985939 (Czech Academy of Sciences) and DG16P02M041 (NAKI II of the Ministry of Culture of the Czech Republic) (AN, JP, KP, KŠ, PP); Invacost grants (ANR) and Foundation BNP Paribas (FC); grant I3757-B29 (Austrian Science Foundation FWF) (FE, BL); grant JE 288/9-2 (Deutsche Forschungsgemeinschaft, DFG), BIBS project grant 01LC1501A-H (German Federal Ministry of Education and Research, BMBF) (CM); CABI Development Fund with contributions from DFID (UK), ACIAR (Australia), and DGIS (NLs) and a JdC-Incorporación contract (IJCI-2017-31733) (PG-M); Helsinki Institute of Sustainability Science and the University of Helsinki (RAC); project "GLANCE", 01 LN1320A (BMBF) (GK); and the Oxford Martin School Oxford Martin Programme for the Illegal Wildlife Trade (DV). This study is a contribution of the Invasion Dynamics Network (InDyNet; DFG grant JE 288/8-1).

\section{References}

Albert C, Luque GM, and Courchamp F. 2018. The twenty most charismatic species. PLoS ONE 13: e0199149.

Allen BL. 2018. And the people's choice award goes to...dogs: a comment on Home et al. Anim Conserv 21: 283-84.

Bacher S, Blackburn TM, Essl F, et al. 2018. Socio-economic impact classification of alien taxa (SEICAT). Methods Ecol Evol 9: 159-68.

Backstrom AC, Garrard GE, Hobbs RJ, et al. 2018. Grappling with the social dimensions of novel ecosystems. Front Ecol Environ 16: 109-17.

Beever EA, Simberloff D, Crowley SL, et al. 2019. Social-ecological mismatches create conservation challenges in introduced species management. Front Ecol Environ 17: 117-25.

Benediktsson K. 2015. Floral hazards: Nootka lupin in Iceland and the complex politics of invasive life. Geogr Ann B 97: 139-54.

Bertolino S and Genovesi P. 2003. Spread and attempted eradication of the grey squirrel (Sciurus carolinensis) in Italy, and consequences for the red squirrel (Sciurus vulgaris) in Eurasia. Biol Conserv 109: 351-58.

Blackburn TM, Pyšek P, Bacher S, et al. 2011. A proposed unified framework for biological invasions. Trends Ecol Evol 26: 333-39.

Byerly H, Balmford A, Ferraro PJ, et al. 2018. Nudging proenvironmental behavior: evidence and opportunities. Front Ecol Environ 16: 159-68.

Chucholl C and Wendler F. 2017. Positive selection of beautiful invaders: long-term persistence and bio-invasion risk of freshwater crayfish in the pet trade. Biol Invasions 19: 197-208.

Clark JA and May RM. 2002. Taxonomic bias in conservation research. Science 297: 191-92.

Clavero M. 2014. Shifting baselines and the conservation of nonnative species. Conserv Biol 28: 1434-36.

Courchamp F, Jarić I, Albert C, et al. 2018. The paradoxical extinction of the most charismatic animals. PLoS Biol 16: e2003997.

Crowley SL, Hinchliffe S, and McDonald RA. 2017. Conflict in invasive species management. Front Ecol Environ 15: 133-41.

Crowley SL, Hinchliffe S, and McDonald RA. 2018. Killing squirrels: exploring motivations and practices of lethal wildlife management. Environ Planning E: Nat Space 1: 120-43.

Crowley SL, Hinchliffe S, and McDonald RA. 2019. The parakeet protectors: understanding opposition to introduced species management. J Environ Manage 229: 120-32. 
Dembitzer J. 2017. The case for hippos in Colombia. Isr J Ecol Evol 63: $5-8$.

Dickie IA, Bennett BM, Burrows LE, et al. 2014. Conflicting values: ecosystem services and invasive tree management. Biol Invasions 16: 705-19.

Donaldson MR, Burnett NJ, Braun DC, et al. 2016. Taxonomic bias and international biodiversity conservation research. Facets 1: 105-13.

Ellis MM and Elphick CS. 2007. Using a stochastic model to examine the ecological, economic and ethical consequences of population control in a charismatic invasive species: mute swans in North America. J Appl Ecol 44: 312-22.

Estévez RA, Anderson CB, Pizarro JC, et al. 2015. Clarifying values, risk perceptions, and attitudes to resolve or avoid social conflicts in invasive species management. Conserv Biol 29: 19-30.

Fischer A, Selge S, van der Wal R, and Larson BMH. 2014. The public and professionals reason similarly about the management of non-native invasive species: a quantitative investigation of the relationship between beliefs and attitudes. PLoS ONE 9: e105495.

Fleming PA and Bateman PW. 2016. The good, the bad, and the ugly: which Australian terrestrial mammal species attract most research? Mammal Rev 46: 241-54.

García-Llorente M, Martín-López B, González JA, et al. 2008. Social perceptions of the impacts and benefits of invasive alien species: implications for management. Biol Conserv 141: 2969-83.

Genovesi P and Bertolino S. 2001. Human dimension aspects in invasive alien species issues: the case of the failure of the grey squirrel eradication project in Italy. In: McNeely JA (Ed). The great reshuffling: human dimensions of invasive alien species. Gland, Switzerland: IUCN.

Gobster PH. 2011. Factors affecting people's responses to invasive species management. In: Rotherham ID and Lambert RA (Eds). Invasive and introduced plants and animals: human perceptions, attitudes and approaches to management. London, UK: Earthscan.

Green SJ, Underwood EB, and Akins JL. 2017. Mobilizing volunteers to sustain local suppression of a global marine invasion. Conserv Lett 10: 726-35.

Ikeda T, Asano M, Yohei Matoba Y, et al. 2004. Present status of invasive alien raccoon and its impact in Japan. Global Environ Res 8: 125-31.

Jarić I, Correia RA, Roberts DL, et al. 2019. On the overlap between scientific and societal taxonomic attentions - insights for conservation. Sci Total Environ 648: 772-78.

Kueffer C and Kull CA. 2017. Non-native species and the aesthetics of nature. In: Montserrat Vilà M and Hulme PE (Eds). Impact of biological invasions on ecosystem services. Berlin, Germany: Springer.

Kutlvašr J, Pergl J, Baroš A, et al. 2019. Survival, dynamics of spread and invasive potential of species in perennial plantations. Biol Invasions 21: 561-73.

Ladle RJ, Correia RA, Do Y, et al. 2016. Conservation culturomics. Front Ecol Environ 14: 270-76.

Larson BM. 2005. The war of the roses: demilitarizing invasion biology. Front Ecol Environ 3: 495-500.

Liordos V, Kontsiotis VJ, Georgari M, et al. 2017. Public acceptance of management methods under different human-wildlife conflict scenarios. Sci Total Environ 579: 685-93.

Lorimer J. 2007. Nonhuman charisma. Environ Plann D 25: 911-32.
Lukas J, Kalinkat G, Kempkes M, et al. 2017. Feral guppies in Germany - a critical evaluation of a citizen science approach as biomonitoring tool. Bull Fish Biol 17: 13-27.

Maceda-Veiga A, Domínguez-Domínguez O, Escribano-Alacid J, et al. 2016. The aquarium hobby: can sinners become saints in freshwater fish conservation? Fish Fish 17: 860-74.

Mack RN. 2001. Motivations and consequences of the human dispersal of plants. In: McNeely JA (Ed). The great reshuffling: human dimensions of invasive alien species. Gland, Switzerland: IUCN.

McNeely JA. 2001. An introduction to human dimensions of invasive alien species. In: McNeely JA (Ed). The great reshuffling: human dimensions of invasive alien species. Gland, Switzerland: IUCN.

Melero Y. 2017. Communication of flagship species in conservation: lessons from invasive management projects. Biodivers Conserv 26: 2973-78.

Novoa A, Shackleton R, Canavan S, et al. 2018. A framework for engaging stakeholders on the management of alien species. $J$ Environ Manage 205: 286-97.

Nuñez MA and Simberloff D. 2005. Invasive species and the cultural keystone species concept. Ecol Soc 10: 4.

Nuñez MA, Kuebbing S, Dimarco RD, et al. 2012. Invasive species: to eat or not to eat, that is the question. Conserv Lett 5: 334-41.

Padilla DK and Williams SL. 2004. Beyond ballast water: aquarium and ornamental trades as sources of invasive species in aquatic ecosystems. Front Ecol Environ 2: 131-38.

Pagès M, Fischer A, and van der Wal R. 2018. The dynamics of volunteer motivations for engaging in the management of invasive plants: insights from a mixed-methods study on Scottish seabird islands. J Environ Plann Man 61: 904-23.

Petursdottir T, Aradottir AL, and Benediktsson K. 2013. An evaluation of the short-term progress of restoration combining ecological assessment and public perception. Restor Ecol 21: 75-85.

Pyšek P and Richardson DM. 2008. Traits associated with invasiveness in alien plants: where do we stand? In: Nentwig W (Ed). Biological invasions. Berlin, Germany: Springer.

Pyšek P, Richardson DM, Pergl J, et al. 2008. Geographical and taxonomic biases in invasion ecology. Trends Ecol Evol 23: 237-44.

Schüttler E, Rozzi R, and Jax K. 2011. Towards a societal discourse on invasive species management: a case study of public perceptions of mink and beavers in Cape Horn. J Nat Conserv 19: 175-84.

Shackleton RT, Richardson DM, Shackleton CM, et al. 2019. Explaining people's perceptions of invasive alien species: a conceptual framework. J Environ Manage 229: 10-26.

Soga M and Gaston KJ. 2018. Shifting baseline syndrome: causes, consequences, and implications. Front Ecol Environ 16: 222-30.

van der Wal R, Fischer A, Selge S, et al. 2015. Neither the public nor experts judge species primarily on their origins. Environ Conserv 42: 349-55.

van Kleunen M, Essl F, Pergl J, et al. 2018. The changing role of ornamental horticulture in alien plant invasions. Biol Rev 93: 1421-37.

Veitch CR and Clout MN. 2001. Human dimensions in the management of invasive species in New Zealand. In: McNeely JA (Ed). The great reshuffling: human dimensions of invasive alien species. Gland, Switzerland: IUCN.

Verbrugge LN, Van den Born RJ, and Lenders HR. 2013. Exploring public perception of non-native species from a visions of nature perspective. Environ Manage 52: 1562-73. 
Veríssimo D, MacMillan DC, and Smith RJ. 2011. Toward a systematic approach for identifying conservation flagships. Conserv Lett 4: $1-8$.

Veríssimo D, Vaughan G, Ridout M, et al. 2017. Increased conservation marketing effort has major fundraising benefits for even the least popular species. Biol Conserv 211: 95-101.

Wallach AD, Bekoff M, Batavia C, et al. 2018. Summoning compassion to address the challenges of conservation. Conserv Biol 32: 1255-65.

Wilson JR, Procheş Ş, Braschler B, et al. 2007. The (bio)diversity of science reflects the interests of society. Front Ecol Environ 5: 409-14.

\section{Supporting Information}

Additional, web-only material may be found in the online version of this article at http://onlinelibrary.wiley.com/doi/10. 1002/fee.2195/suppinfo
University of Helsinki, Helsinki, Finland; ${ }^{4}$ Environment and Sustainability Institute, University of Exeter, Penryn, UK; ${ }^{5}$ Department of Botany and Biodiversity Research, University of Vienna, Vienna, Austria; ${ }^{6}$ Division of Environmental Communication, Swedish University of Agricultural Sciences, Uppsala, Sweden; ${ }^{7} C A B I$, Egham, UK; ${ }^{8}$ RNM360, Departamento de Ingeniería Forestal, Universidad de Córdoba, Córdoba, Spain; ${ }^{9}$ Leibniz-Institute of Freshwater Ecology and Inland Fisheries, Berlin, Germany; ${ }^{10}$ School of Biological Sciences, University of Aberdeen, Aberdeen, UK; ${ }^{11}$ Université Paris Dauphine, PSL Research University, CNRS, Paris, France; ${ }^{12}$ Modelling, Evidence and Policy Group, Newcastle University, Newcastle, UK; ${ }^{13}$ Institute of Biology, Freie Universität Berlin, Berlin, Germany; ${ }^{14}$ Czech Academy of Sciences, Institute of Botany, Department of Invasion Ecology, Průhonice, Czech Republic;

${ }^{15}$ Department of Ecology, Faculty of Science, Charles University, Prague, Czech Republic; ${ }^{16}$ West Iceland Nature Research Centre, Stykkishólmur, Iceland; ${ }^{17}$ Institute of Geography and Sustainability, University of Lausanne, Lausanne, Switzerland; ${ }^{18}$ Department of Zoology, University of Oxford, Oxford, UK; ${ }^{19}$ Institute for Conservation Research, San Diego Zoo Global, San Diego, CA

\section{FrontiersEcoPics}

\section{Mysterious beetle and butterfly aggregation}

D uring fieldwork near Lake Bleu, in the El Kala region of northeastern Algeria, we encountered an unfamiliar interaction between the speckled rose chafer beetle (Protaetia morio) and the two-tailed pasha butterfly (Charaxes jasius). Both species are common in the Mediterranean.

Groups of around six to eight beetles were aggregating on branch crotches of a strawberry tree (Arbutus unedo). These groups were frequently joined by one or two butterflies (https://bit.ly/3eSXHu1). We noticed that the butterflies did not land near single beetles, and from their flight patterns, they seemed to know exactly where the beetles were, as if they were attracted by chemicals.

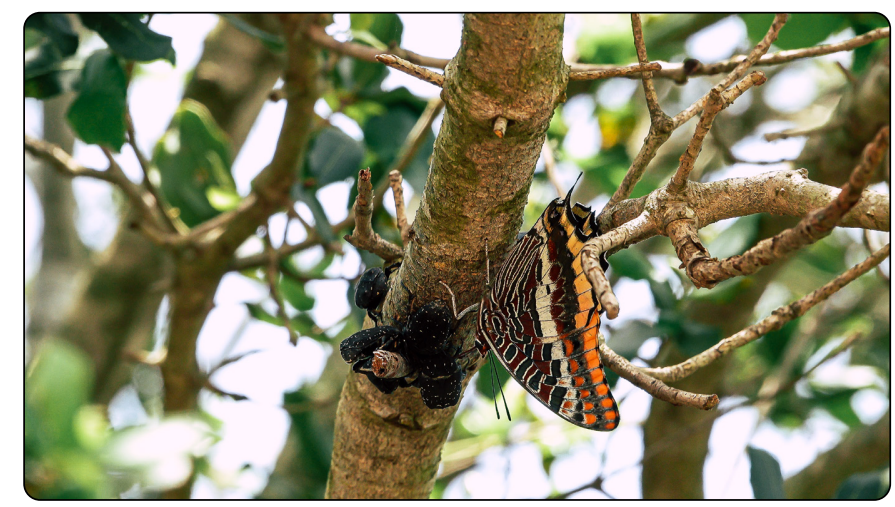

We observed the butterflies trying to feed on something in the middle of the beetle groups. Because the two species have similar diets (nectar, ripe fruit, and tree sap), our first impression was that the butterflies were consuming tree sap in order to obtain water, amino acids, and minerals. However, we closely examined different branch crotches where the beetles congregated and found no signs of an exudate or wounds. Neither the beetle nor the butterfly has the ability to extract xylem sap by itself. Could the butterfly have been feeding on the excretions of the beetle? Why do the beetle and butterfly target branch crotches? Does this interaction involve a more complicated interspecific chemical communication between the plant and the two insect species? Future field surveys and experiments may shed light on this mystery.

Rassim Khelifa ${ }^{1}$, Rabah Zebsa ${ }^{2}$, and Hayat Mahdjoub ${ }^{2}$ ${ }^{1}$ University of British Columbia, Vancouver, Canada; ${ }^{2}$ Université de Guelma, Guelma, Algeria doi:10.1002/fee.2238

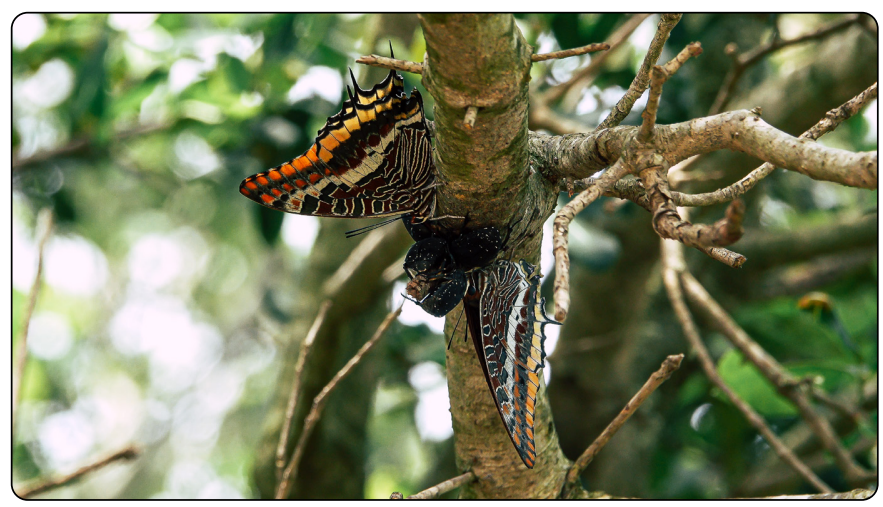

